\title{
Immunohistochemical Expression of Ki-67, p53 and HER2/neu in Meningiomas
}

\author{
Aijaz Muzamil Dar ${ }^{1}$, Nisha TG $^{2 *}$, Prema Saldanha ${ }^{3}$, Ksheera Cariappa $^{4}$ \\ ${ }^{1}$ Senior Resident, Department of Pathology, Sher-I-Kashmir Institute of Medical Sciences, Soura, Srinagar, Kashmir, India. \\ ${ }^{2}$ Assistant Professor, Department of Pathology, Yenepoya Medical College, Deralakatte, Mangalore, India \\ ${ }^{3}$ Professor, Department of Pathology, Yenepoya Medical College, Deralakatte, Mangalore, India \\ ${ }^{4}$ Oncopathology Fellow Resident, Department of Pathology, Yenepoya Medical College, Deralakatte, Mangalore, India
}

\begin{abstract}
DOI: $\underline{10.36348 / \text { sjpm.2020.v05i03.001 }}$
| Received: 22.02.2020 | Accepted: 02.03.2020 | Published: 07.03.2020

*Corresponding author: Nisha T G
\end{abstract}

\section{Abstract}

Introduction: Meningiomas are one of the most common primary intracranial tumours. Although most meningiomas are benign, the spectrum also includes atypical and malignant meningiomas. Clinical outcome of meningiomas is often difficult to predict. The stratification of risk on the basis of histomorphology alone remains problematic; thus additional biomarkers are needed. In this study biomarkers of prognostic and therapeutic interest like Ki-67, p53 and HER2/neu have been studied in correlation with clinicopathological parameters. Materials and methods: A retrospective study on histologically diagnosed meningiomas was undertaken. Slides were retrieved and reviewed. Clinical details were recorded from the files in the archives of the department. Immunohistochemical staining with markers Ki-67, p53 and HER2/neu were performed and the findings were interpreted. Results: The study included 17 cases with an age range of 16 to 73 years and a male: female ratio of $1.1: 1$. There were 11 cases $(64.7 \%)$ of primary tumours and 6 cases $(35.3 \%)$ were recurrent tumours. WHO Grade I meningiomas were maximum and accounted for $70.5 \%$ followed by grade II $(17.5 \%)$ and grade III (12\%) meningiomas. Ki-67 expression was seen in all cases with progressively increased expression in higher grades. p53 expression was observed in all the cases with higher levels (>10\%) in Grade II and Grade III meningiomas compared to grade I meningiomas. The HER2/neu staining was negative in all cases studied. Conclusion: Grade I meningiomas appear to have low mitotic count on morphology but have higher proliferation rate on Ki-67 studies. Most of the recurrent tumours have higher p53 expression (>10\%). Hence, adjuvant studies with biomarkers Ki-67 and p53 will be helpful in precise grading of meningiomas.

Keywords: Meningioma, Ki-67, p53, HER2/neu, WHO tumour grade.

Copyright @ 2020: This is an open-access article distributed under the terms of the Creative Commons Attribution license which permits unrestricted use, distribution, and reproduction in any medium for non-commercial use (NonCommercial, or CC-BY-NC) provided the original author and sources are credited.

\section{INTRODUCTION}

Meningiomas are common tumours of central nervous system (CNS), accounting for $15 \%$ to $25 \%$ of primary intracranial and intraspinal neoplasms. These are thought to arise from the cells of the arachnoid membrane covering the brain and spinal cord [1]. The peak incidence is found between the sixth and seventh decades of life, affecting females more often than males (2:1) [2]. Most of the patients present with neurological signs and symptoms, due to compression of the adjacent structures by the tumour. Radiological imaging techniques have limited ability to differentiate between different subtypes of meningiomas [3].

According to World Health Organization (WHO) meningiomas are classified as benign, atypical and malignant/anaplastic types. About $80 \%$ of meningiomas are slow-growing benign tumours of WHO grade I with many histological types. Complete resection of benign meningiomas (Grade I) is associated with 5 -year recurrence rates of only $5 \%$, whereas in the atypical meningiomas (GradeII), it has been found to be high (40\%). Anaplastic meningiomas (Grade III) account for $1-3 \%$ of all meningiomas and have clinical characteristics similar to other malignant neoplasms [4].

Morphological parameters such as histological type, WHO grade, mitotic counts and brain invasion have been found to have prognostic value [5]. Histologically distinct subsets of meningiomas are associated with high risk of recurrence even after complete resection. The progression of meningiomas is difficult to predict clinically and the risk of such progression has been assessed mainly on histomorphological parameters with interobserver 
variability. Currently there are only a few ancilliary techniques that are helpful in predicting the recurrence and prognostic behaviour of meningiomas [1]. The present study was undertaken to analyse the immunohistochemical expression status of Ki-67, p53 and HER2/neu in meningiomas in correlation with pathological parameters like WHO tumour grade and histological subtype.

\section{MATERIALS AND METHODS}

A retrospective study was conducted in the department of Pathology attached to a tertiary care hospital. The haematoxylin and eosin $(\mathrm{H} \& \mathrm{E})$ stained slides were retrieved and reviewed. All relevant clinical details were recorded from the files available in the archives of the department. Meningiomas were classified into three groups, according to WHO classification and diagnostic criteria as follows [6]: classic meningiomas (WHO grade I), atypical meningiomas (WHO grade II), and anaplastic meningiomas (WHO grade III). Atypical meningiomas were defined as those with either a high mitotic rate ( $>4$ per $10 \mathrm{HPFs}$ ) or the presence of at least 3 of these 4 features: small cell formation, macronucleoli, sheeting architecture, and hypercellularity. Anaplastic meningiomas were defined as those with either a high mitotic count (>20 per $10 \mathrm{HPFs}$ ) or with histologic features similar to those of carcinoma, sarcoma, and melanoma focally or diffusely $[1,6]$.

The best paraffin embedded block was selected and standard 4 micron sections were subjected to immunohistochemical study with appropriate positive and negative controls. Primary antibodies against Ki-67 (a mouse monoclonal antibody of GM001 clone, Pathnsitu), p53 (mouse monoclonal antibody of BP-53-12 clone, Pathnsitu) and HER2/neu (a rabbit monoclonal antibody of EP3 clone, Pathnsitu) were used. The Polyexcel HRP (non-biotin, micro-polymer based) /DAB Detection system was followed.

\section{Interpretation of Immunohistochemical (IHC) result}

Ki67 expression in tumour cells with brown nuclear staining was considered as positive. The number of positive cells in 10 high power fields (HPF) was observed. The scoring was done as score $1(<4$ positive cells/ 10HPF), 2 (4-19 positive cells/ 10HPF) and 3 ( $\geq 20$ positive cells/ 10HPF) [6]. p53 expression in tumour cells with brown nuclear staining was considered positive and calculated as percentage of positive cells, by counting at least 1000 tumor cells in fields with the largest number of positive cells. The expression status was grouped into low $(<10 \%)$ and high $(\geq 10 \%)[4]$.

HER2/neu immunostaining is not usually found within arachnoid cells of normal meninges, hence its expression was always considered as an overexpression [1]. HER2/neu scoring was done similar to the breast carcinoma scoring system [5]. Score 0 (negative) - no staining observed or incomplete or barely perceptible membrane staining within $\leq 10 \%$ of tumour cells; $1+($ negative $)$ - incomplete membrane staining that is faintly/barely perceptible within $\geq 10 \%$ of tumour cells; 2+(equivocal) - incomplete and /or weak /moderate circumferential membrane staining within $>10 \%$ of tumour cells or intense complete and circumferential membrane staining within $\leq 10 \%$ of tumour cells; 3+(positive) - complete and intense circumferential membrane staining within $>10 \%$ of tumour cells. Scores of 0 or $1+$ were considered negative for HER2/neu expression, scores of $2+$ was equivocal and $3+$ as positive for HER2/neu expression [5].

\section{RESULTS}

During the study period, 17 cases of meningiomas were found, out of which $11(64.7 \%)$ cases were primary tumours and $6(35.3 \%)$ cases were recurrent tumours. Summary of meningioma cases studied are shown in Table 1. The age of the patients ranged from 16 to 73 years, with a mean age of 46.41 years. A total of $9(53 \%)$ patients were males and 8 $(47 \%)$ patients were females with a male: female ratio of 1.1:1. Parietal region was the common location in this study. Grade I meningiomas accounted for $70.5 \%$, grade II $(17.5 \%)$ and grade III $(12 \%)$ of the studied cases. In grade I meningiomas, the most common histologic type was meningotheliomatous 8 cases (66.6\%), followed by transitional (16.6\%) and psammomatous meningiomas (16.6\%). In grade II meningiomas, both the cases were atypical meningiomas. Among the Grade III meningiomas there was one case each of rhabdoid and anaplastic meningioma. Photomicrographs of meningothelial and psammomamatous meningiomas shown in Figure 1 and 2. 
Table-1: Summary of meningioma cases studied

\begin{tabular}{|l|l|l|l|l|l|l|l|l|}
\hline $\begin{array}{c}\text { Sl } \\
\text { No }\end{array}$ & $\begin{array}{c}\text { Age } \\
(\mathbf{Y r s})\end{array}$ & Sex & $\begin{array}{c}\text { WHO } \\
\text { Grade of } \\
\text { tumour }\end{array}$ & $\begin{array}{c}\text { Histological type of } \\
\text { meningioma }\end{array}$ & $\begin{array}{c}\text { Primary or } \\
\text { recurrent } \\
\text { tumor }\end{array}$ & $\begin{array}{c}\text { Ki-67 } \\
\text { Score }\end{array}$ & $\begin{array}{c}\text { p53 } \\
\text { Score }\end{array}$ & $\begin{array}{c}\text { HER2/neu } \\
\text { Score }\end{array}$ \\
\hline 1 & 48 & F & I & Transitional & Primary & 1 & Low & 0 \\
\hline 2 & 57 & F & I & Transitional & Primary & 2 & High & 0 \\
\hline 3 & 53 & M & I & Psammomatous & Primary & 2 & Low & 0 \\
\hline 4 & 57 & M & I & Meningothelial & Primary & 2 & High & 0 \\
\hline 5 & 39 & F & I & Meningothelial & Primary & 2 & Low & 0 \\
\hline 6 & 72 & F & I & Psammomatous & Primary & 3 & High & 0 \\
\hline 7 & 26 & F & I & Meningothelial & Primary & 3 & High & 0 \\
\hline 8 & 34 & F & I & Meningothelial & Primary & 3 & High & 0 \\
\hline 9 & 66 & M & I & Meningothelial & Primary & 3 & High & 0 \\
\hline 10 & 36 & M & II & Atypical & Primary & 3 & High & 0 \\
\hline 11 & 50 & M & III & Rhabdoid & Primary & 3 & High & 0 \\
\hline 12 & 32 & M & I & Meningothelial & Recurrent & 1 & High & 0 \\
\hline 13 & 42 & F & I & Meningothelial & Recurrent & 2 & Low & 0 \\
\hline 14 & 53 & M & I & Meningothelial & Recurrent & 2 & High & 0 \\
\hline 15 & 35 & M & II & Atypical & Recurrent & 3 & High & 0 \\
\hline 16 & 73 & M & II & Atypical & Recurrent & 3 & High & 0 \\
\hline 17 & 16 & F & III & Malignant & Recurrent & 2 & High & 0 \\
\hline
\end{tabular}

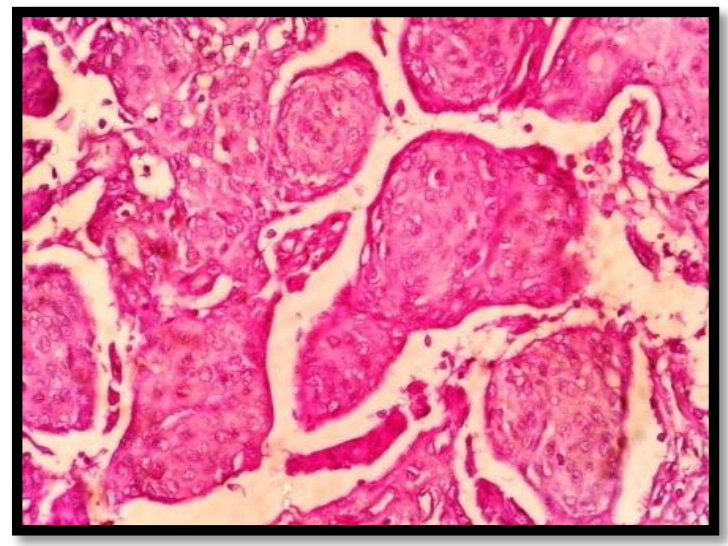

Fig-1: Showing meningothelial meningioma. (H\&E, 10x)

The Ki-67 expression was observed in all cases of meningiomas. In the primary grade I meningiomas ( $9 / 11$ cases) there were one case of score 1, 4 cases each of score 2 and score 3 on Ki-67 study. One case each of primary grade 2 and grade 3 meningiomas showed score 3 on Ki-67 study. There were 3 recurrent grade I meningiomas wherein one

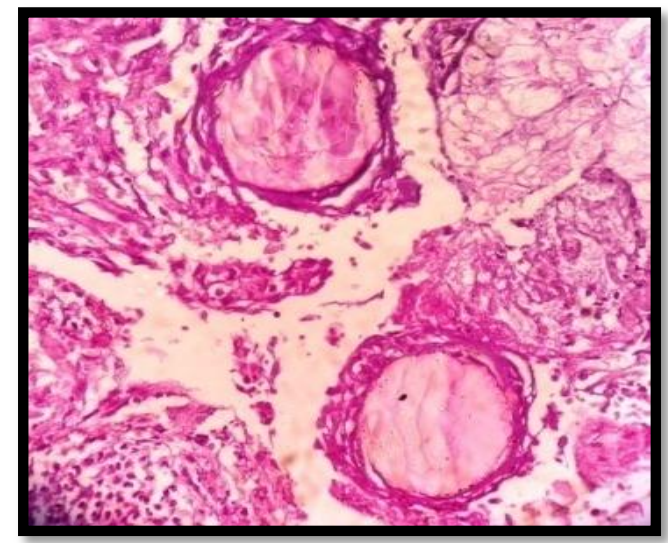

Fig-2: Showing psammomma bodies in meningioma. (H\&E, 10x)

case had score 1 and two cases score 2. The 2 cases of recurrent grade 2 meningioma showed score 3 and one case of recurrent grade 3 meningioma showed score 2 on Ki-67 labelling. The Ki-67 expression of various grades of meningiomas with their score is shown in Table 2. Photomicrographs of different scores of Ki-67 on IHC given in Figure 3.

Table-2: Ki-67 expression in primary and recurrent tumours in correlation with WHO grade

\begin{tabular}{|l|l|c|c|c|}
\hline \multirow{2}{*}{ WHO Grade } & & \multicolumn{3}{|c|}{ Ki 67 Expression } \\
\cline { 2 - 5 } & & Score 1 & Score 2 & Score 3 \\
\hline \multirow{3}{*}{$\begin{array}{c}\text { Primary } \\
\text { Tumours } \\
(\mathrm{n}=11)\end{array}$} & Grade I (9) & 1 & 4 & 4 \\
\cline { 2 - 5 } & Grade II (1) & - & - & 1 \\
\cline { 2 - 5 } $\begin{array}{c}\text { Recurrent } \\
\text { Tumours } \\
(\mathrm{n}=6)\end{array}$ & Grade III (1) & - & - & - \\
\cline { 2 - 5 } & Grade I (3) & 1 & 2 & - \\
\cline { 2 - 5 } & Grade II (2) & - & - & - \\
\hline
\end{tabular}




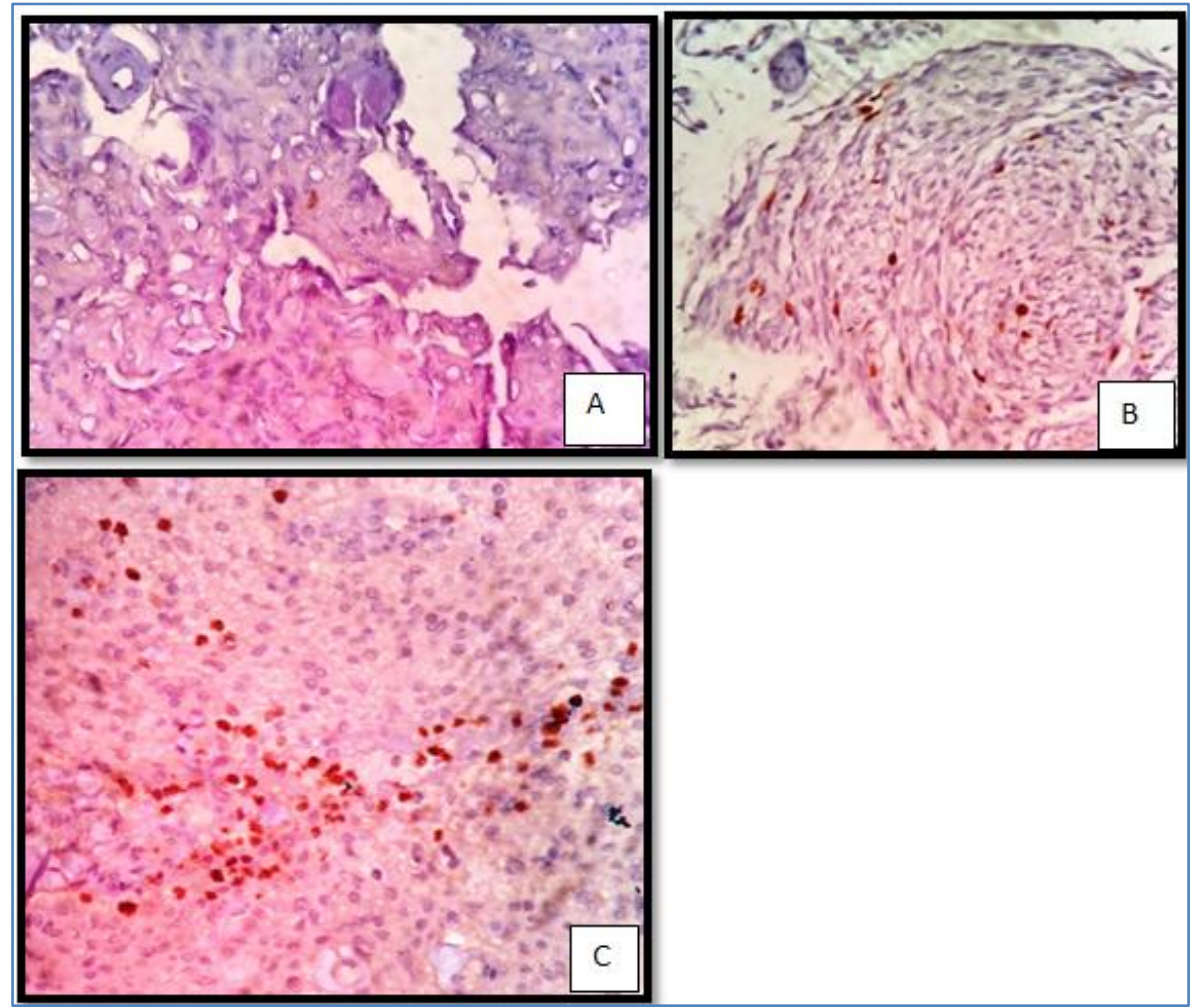

Fig-3: IHC with Ki-67 showing score 1 (A), score 2 (B), score 3 staining (C).

The p53 expression was scored as low and high expression. Out of the 9 primary grades I meningiomas 3 cases showed low expression and other 6 cases showed high expression. One case each of primary grade 2 and 3 meningiomas showed high p53 expression. In recurrent tumours, out of 3 grade 1 meningiomas, 2 cases had high p53 expression. All the grade 2 and grade 3 recurrent meningiomas showed high p53 expression. The p53 expression of various grades of meningiomas with their score is shown in Table 3. Photomicrographs of p53 score given in Figure 4.

Table-3: P53 expression in primary and recurrent tumours in comparison to WHO grade

\begin{tabular}{|l|l|c|c|}
\hline WHO grade & & \multicolumn{2}{|c|}{ p53 Expression } \\
\cline { 2 - 4 } & & Low (<10\%) & High (>10\%) \\
\hline \multirow{3}{*}{$\begin{array}{c}\text { Primary Tumour } \\
\mathbf{n}=\mathbf{1 1}\end{array}$} & Grade I (9) & 3 & 6 \\
\cline { 2 - 4 } & Grade II (1) & - & 1 \\
\cline { 2 - 4 } $\begin{array}{c}\text { Recurrent } \\
\text { Tumour } \\
\mathbf{n}=\mathbf{6}\end{array}$ & Grade III (1) & - & 1 \\
\cline { 2 - 4 } & Grade I (3) & 1 & 2 \\
\cline { 2 - 4 } & Grade II (2) & - & 1 \\
\hline
\end{tabular}

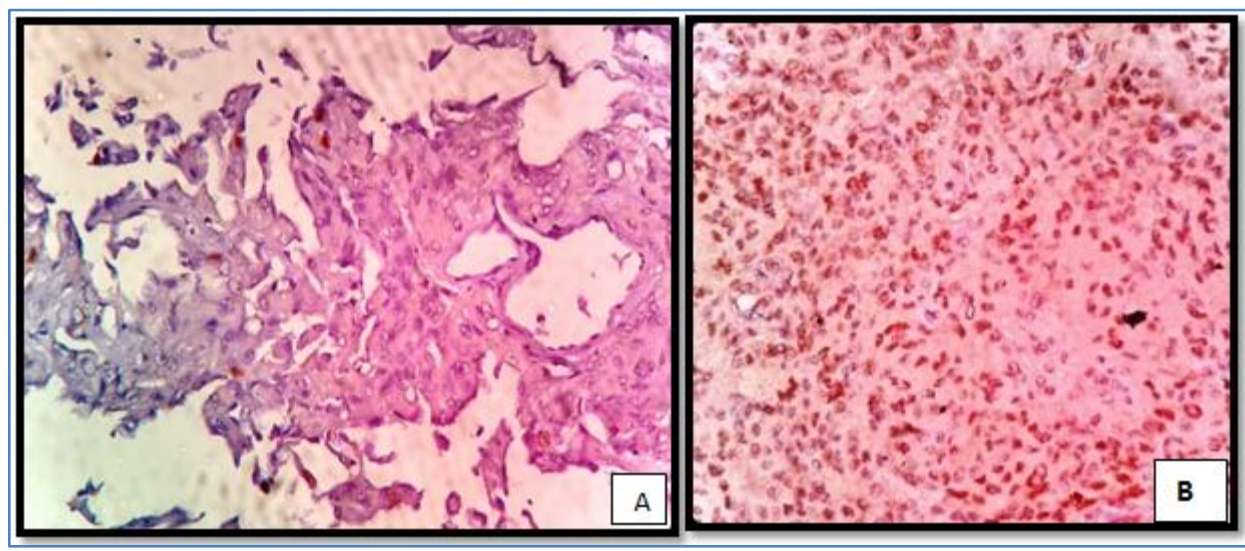

Fig-4: IHC with p53 showing low expression $(<10 \%)$ in $A$ and high expression $(\geq 10 \%)$ in $B$ 
The HER2/neu expression in all the 17 cases did not show membranous positivity and were considered negative for HER2/neu (grade 0). Only faint cytoplasmic positivity was seen in some cases which were considered negative (Figure 5).

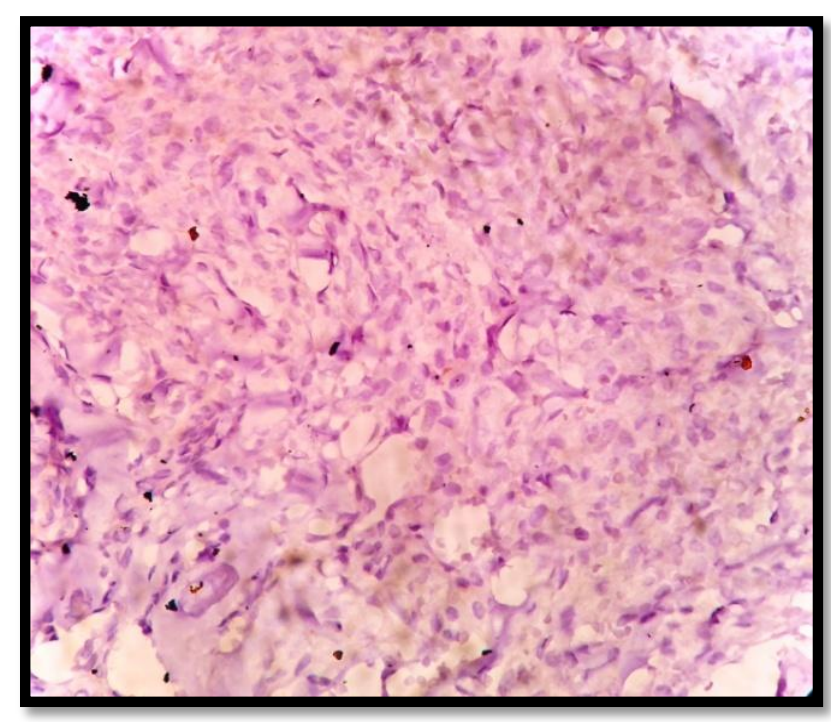

Fig-5: IHC with HER2/neu showing negative staining (score 0)

\section{DISCUSSION}

Meningiomas are one of the most common primary brain tumours with many morphological variants. WHO grade I tumors include histological types like meningothelial, fibrous, transitional, psammomatous, angiomatous, microcystic, secretory, lymphoplasmacytic rich and metaplastic meningiomas. Distinct subtypes like chondroid and clear cell meningiomas are classified as WHO grade II and the papillary and rhabdoid meningiomas are WHO grade III meningiomas [4]. There are morphological criteria that define different grades of meningioma; however, the distinction by morphology alone is difficult. There has been no highly reliable immunohistochemical marker that can separate the different WHO grades till date.

The differentiation between grade I and grade II meningiomas at times with assessment of mitotic figures alone would lead to interobserver variability [4]. Atypical and anaplastic meningiomas with active proliferation behave more aggressively with higher tendency for recurrence [4]. However, there exist a borderline and a group of cases that show clinically aggressive behavior despite being histologically benign (WHO Grade I). As many as 7-20\% of these benign (WHO Grade I) tumours are known to recur [7]. In the recent years, interest in this clinically diverse group of tumours has intensified, leading researchers to investigate combinations of predictive and prognostic factors, with the aim to identify the patients who should be followed more closely for recurrence or treated more aggressively at the time of diagnosis [8].
In this context, adjuvant biomarkers are needed to facilitate the assessment of proliferation accurately. Thus, this study was undertaken to detect the immunohistochemical expression of Ki-67, p53 and HER2/neu in WHO grades I-III meningiomas.

In the present study the age of the patients ranged from 16 to 73 years, with a mean age of 46.41 years which was consistent with other studies $[9,10]$. Our results were in accordance with the common agreement that most meningiomas are benign and grade I meningiomas constituted the majority of the tumours accounting for $70.5 \%$ of the studied cases [11]. With the tumour histological subtype, the most common subtype in the current study was meningothelial $(66.6 \%)$, followed by transitional (16.6\%) and psammomatous meningioma (16.6\%). These findings fall within the figures given by the WHO classification of tumours of the central nervous system [6, 11]. Though recurrence is known to be more common in Grade II-III meningiomas, the present study had 3 cases $(50 \%)$ of grade I meningiomas in the ones which had recurred. There was no significant relationship found between the tumour histological subtype and tumour grade, a finding that correlated with a study which highlighted that even though a vast range of meningioma subtypes exist, the clinical behavior and outcomes correlate with the WHO grade, and not on the histologic subtype [11].

Finding of atypia on histology is highly subjective, with significant interobserver variability. Thus an objective method of counting positively stained nuclei on IHC provides a more reproducible and an accurate method for assessing aggressive behavior as an adjunct to histology [7]. MIB-1 is an anti-Ki-67 monoclonal antibody that has been found to be useful for the analysis of proliferative potential [11]. This has been widely used in many studies of meningiomas as a prognostic marker and adjuvant to histopathology for grading of meningiomas. A high $\mathrm{Ki}-67$ index is associated with aggressiveness and poor prognosis for meningiomas [12]. Thus tumours that do not have any histological features of malignancy but have a high score should be reviewed carefully [7]. Precaution should be taken in interpreting Ki-67 positivity on IHC, as lymphocytes or other proliferative cells can show positivity for Ki-67 antigen. This error can be eliminated by meticulously comparing with the hematoxylin and eosin section ( $\mathrm{H}$ and $\mathrm{E})$. This reinforces the fact that IHC stains can only be used as an adjunct to histology and cannot replace them [13].

Various studied in the past have shown that proliferative index increases with the increasing tumour grade [13]. In this study $\mathrm{Ki}-67$ marker was positive in all the cases. The cases of primary grade II and III meningiomas all revealed higher Ki-67 expression. Interestingly, even some of the primary grade I tumours showed higher Ki-67 scores of 2 and 3 which suggest 
that though these grade 1 tumours may have low mitotic count in routine $\mathrm{H} \& \mathrm{E}$ sections but they might have high proliferation rate which can be detected by Ki-67 antigen as IHC marker. Similar finding of morphologically benign tumours with high proliferative index was also noted in another study [13]. The morphological grading of meningiomas based on mitotic count alone is highly subjective, leading to low accuracy. Hence, Ki-67 can be used as routine IHC marker in all meningioma cases to categorise patients who has more chance of recurrence which helps in individualising the therapy for meningioma patients [7].

The p53 protein functions as a tumour suppressor wherein following irreversible DNA damage, the p53 protein induces cell apoptosis. The wild-type protein has an inhibitory effect on cell proliferation and transformation, but gene mutations alter its tumour suppressor activity. A high proportion of cells with mutant protein indicate increased tumour aggressiveness [2]. The positive p53 expression has been reported in a range of $10-88 \%$ [4]. One study reported an immunoreactivity for p53 (9.5\% grade I, $72.7 \%$ grade II, and $88.9 \%$ grade III meningiomas), and also found increasing expression levels with higher grades [14]. In our study, only high p53 expression was observed in all the cases with higher levels in Grade II and Grade III meningiomas compared to grade I meningiomas which is consistent with other studies [14]. However, in another large study done, there was no significant association between p53 levels and tumour grade [4]. In our study out of 9 primary grade I meningiomas, 6 cases showed high expression and in recurrent tumours, out of 3 grade1 meningiomas, 2 cases had high p53 expression. Thus, grade I meningiomas can show higher p53 expression which may indicate aggressive behaviour of the tumour. High Ki-67 proliferative activity and p53 expression in recurrent cases was observed in this study which was consistent with the study done by Rao et al. [13].

The targeted therapy for HER2/neu is proving to be effective; researchers in the last decade have shown interest in the role of HER2/neu as a possible target for therapy in meningiomas also [15]. The HER2/neu expression in meningiomas is observed to be in a wide range of 2.5 to $67.3 \%$ across various studies [1, 16-18]. Although some investigators have refuted any association between HER2/neu expression and the recurrence of meningioma [16], others suggested that it was associated with a poor prognosis $[1,19]$. However, still other investigators concluded that the role of HER2/neu expression in the pathogenesis, progression, and treatment of meningiomas still needs investigation [5], and recommended that investigations should include a combination of biomarkers [18]. In the present study, HER2/neu was negative in all the cases studied. It could be due to wide variation in expression of the marker in different study population [11].

\section{CONCLUSION}

Though the morphological assessment of Grade I meningiomas appears to have low mitotic count, Ki-67 study can show higher proliferation rate. Hence precise assessment of $\mathrm{Ki}-67$ needs to be done and should be included in diagnostic criteria as many of the cases seem to be "on the fence" with regard to tumour grade. Tumours with higher p53 expression levels $(>10 \%)$ have been associated with higher grades, and recurrent tumours. Thus, we suggest that biomarkers Ki67 and p53 should be studied in all cases of meningiomas to improve the accuracy of the grading and to help in categorising higher risk patients thus formulating alternative individualised treatment choices for them.

\section{REFERENCES}

1. Loussouarn, D., Brunon, J., Avet-Loiseau, H., Campone, M., \& Mosnier, J. F. (2006). Prognostic value of HER2 expression in meningiomas: an immunohistochemical and fluorescence in situ hybridization study. Human Pathology, 37(4), 415421.

2. Trott, G., Pereira-Lima, J. F. S., Leães, C. G. S., Ferreira, N. P., Barbosa-Coutinho, L. M., \& Oliveira, M. C. (2015). Abundant immunohistochemical expression of dopamine D2 receptor and p53 protein in meningiomas: followup, relation to gender, age, tumour grade, and recurrence. Brazilian Journal of Medical and Biological Research, 48(5), 415-419.

3. Bhagyalakshmi, A., Prasad, S.V., Swarajyalakshmi, K.V., Parvathi, G., Reddy, B.S., Kartheek, B.V.S. (2020). Clinico-Histopathological Study of Meningiomas in Correlation with Proliferative Index Ki-67. Saudi Journal of Pathology and Microbiology, 5(2), 80-85.

4. Pavelin, S., Becic, K., Forempoher, G., Mrklic, I., Pogorelic, Z., Titlic, M., \& Andelinovic, S. (2013). Expression of $\mathrm{Ki}-67$ and p53 in meningiomas. Neoplasma, 60(5), 480-5.

5. Onak Kandemir, N., Ege Gul, A., Dogan Gun, B., Karadayi, N., Yurdakan, G., \& Ozdamar, S. (2009). Her-2/neu, Estrogen and Progesterone Receptor Expression in WHO Grade I Meningiomas. Medical Journal of Trakya University, 27(3):292-296.

6. Louis, D.N., Ohgaki, H., Wiestier, O.D., Cavenee, W.K., Ellison, D.W., Figarella-Branger, D. (2016). WHO Classification of Tumours of the Central Nervous System. In: Hiroko DNL, Otmar DO. Webster KW editors. 4th Edition. France: International Agency for Research on Cancer.

7. Devaprasath, A., \& Chacko, G. (2003). Diagnostic validity of the Ki-67 labeling index using the MIB1 monoclonal antibody in the grading of meningiomas. Neurology India, 51(3), 336-340.

8. Campbell, B. A., Jhamb, A., Maguire, J. A., Toyota, B., \& Ma, R. (2009). Meningiomas in 2009: controversies and future 
challenges. American Journal of Clinical Oncology, 32(1), 73-85.

9. Kantha, R., Saffari, H. M., \& Suryati, M. Y. (2007). The relationship of p53 protein in meninigioma grading and their various influencing factors amongst neurosurgical patients in Hospital Kuala Lumpur. Medical Journal of Malaysia, 62(3), 194.

10. Csonka, T., Murnyák, B., Szepesi, R., Kurucz, A., Klekner, Á., \& Hortobágyi, T. (2014). Poly (ADPribose) polymerase-1 (PARP1) and p53 labelling index correlates with tumour grade in meningiomas. Folia Neuropathologica, 2, 111-120.

11. Khamis, N. N., \& Rashwan, M. F. (2011). Prognostic role of Her2/neu, progesterone receptor, and MIB-1 expression in meningioma. Egyptian Journal of Pathology, 31(2), 104-112.

12. Lanzafame, S., Torrisi, A., Barbagallo, G., Emmanuele, C., Alberio, N., \& Albanese, V. (2000). Correlation between histological grade, MIB-1, p53, and recurrence in 69 completely resected primary intracranial meningiomas with a 6 year mean follow-up. Pathology-Research and Practice, 196(7), 483-488.

13. Rao, S., Sadiya, N., Doraiswami, S., \& Prathiba, D. (2009). Characterization of morphologically benign biologically aggressive meningiomas. Neurology India, 57(6), 744.

14. Cho, H., Ha, S. Y., Park, S. H., Park, K., \& Chae, Y. S. (1999). Role of p53 gene mutation in tumour aggressiveness of intracranial meningiomas. $J$ Korean Med Sci, 14(2), 199-205.

15. Custer, B. S., Koepsell, T. D., \& Mueller, B. A. (2002). The association between breast carcinoma and meningioma in women. Cancer, 94(6), 16261635.

16. Potti, A., Forseen, S. E., Koka, V. K., Pervez, H., Koch, M., Fraiman, G., \& Levitt, R. (2004). Determination of HER-2/neu overexpression and clinical predictors of survival in a cohort of 347 patients with primary malignant brain tumours. Cancer investigation, 22(4), 537-544.

17. Durand, A., Champier, J., Jouvet, A., Labrousse, F., Honnorat, J., Guyotat, J., \& Fèvre-Montange, M. (2008). Expression of c-Myc, neurofibromatosis Type 2, somatostatin receptor 2 and erb-B2 in human meningiomas: relation to grades or histotypes. Clinical Neuropathology,27(5), 334345 .

18. Wang, C. L., Mei, J. H., Wang, S. S., Xu, S., Xu, L. L., \& Xiong, Y. F. (2010). Expression of HER2/neu in meningiomas: an immunohistochemistry and fluorescence in situ hybridization study. Zhonghua bing li xue za zhi= Chinese journal of pathology, 39(3), 156-160.

19. Amplifikasyonu, G. (2009). Her-2/neu gene amplification in paraffin-embedded tissue sections of meningioma patients. Turkish Neurosurgery, 19(2), 135-138. 